EPJ Web of Conferences 53, 10001 (2013)

DOI: $10.1051 /$ epjconf/20135310001

(C) Owned by the authors, published by EDP Sciences, 2013

\title{
On the energy deposition by electrons in air and the accurate determination of the air-fluorescence yield
}

\author{
J. Rosado ${ }^{a}$, P. Gallego, D. García-Pinto, F. Blanco and F. Arqueros \\ Departamento de Física Atómica, Molecular y Nuclear, Facultad de Ciencias Físicas, \\ Universidad Complutense de Madrid, E-28040, Spain
}

\begin{abstract}
The uncertainty in the absolute value of the air-fluorescence yield still puts a severe limit on the accuracy in the primary energy of ultra-high-energy cosmic rays. The precise measurement of this parameter in laboratory is in turn conditioned by a careful evaluation of the energy deposited in the experimental collision chamber. In this work we discuss on the calculation of the energy deposition and its accuracy. Results from an upgraded Monte Carlo algorithm that we have developed are compared with those obtained using Geant4, showing excellent agreement. These updated calculations of energy deposition are used to apply some corrections to the available measurements of the absolute fluorescence yield, allowing us to obtain a reliable world average of this important parameter.
\end{abstract}

\section{INTRODUCTION}

The fluorescence technique for detection of extensive air showers has been proved to be very fruitful. It is based on the measurement of the faint fluorescence radiation of molecular nitrogen in the $\sim 300-400 \mathrm{~nm}$ spectral range induced by charged particles (mostly electrons) of a shower. By means of imaging telescopes, the longitudinal profile of the shower is recorded allowing its geometrical reconstruction. In addition, it provides a nearly calorimetric measure of the energy of the primary particle by means of the air-fluorescence yield $Y$, that is, the number of photons per unit deposited energy. This key parameter is a function of wavelength and depends on the atmospheric parameters, i.e., pressure, temperature and air composition. Nonetheless the energy scale of fluorescence telescopes is mainly determined by its absolute value, usually given at standard atmospheric conditions (e.g., dry air at $1013 \mathrm{hPa}$ and $293 \dot{\mathrm{K}}$ ) and for the most intense band of the spectrum, located at 337nm.

The air-fluorescence yield is measured in dedicated experiments in laboratory using electron beams to excite air at known conditions in a collision chamber. The induced fluorescence is registered by an appropriate optical system. Several experiments use electrons from a ${ }^{90} \mathrm{Sr}^{-90} \mathrm{Y}$ radioactive source (average energy around $1 \mathrm{MeV}$ ), whereas other experiments use beams from accelerators or electron guns (energies ranging from $\mathrm{keV}$ to $\mathrm{GeV}$ ). It has been shown that the fluorescence yield is nearly independent of electron energy [1-3], which is the underlying principle of a calorimetric determination of the primary energy of extensive air showers. In fact, it is also expected to be independent of the type of incident particle, since both fluorescence emission and energy deposition are governed by low-energy secondary electrons produced in ionization processes.

An accurate experimental determination of the absolute air-fluorescence yield requires an end-toend calibration of the optical system as well as a careful Monte Carlo (MC) evaluation of the energy deposited in the field of view. In this work we discuss on the calculation of the energy deposition and

\footnotetext{
${ }^{a}$ e-mail: jaime_ros@fis.ucm.es
}

This is an Open Access article distributed under the terms of the Creative Commons Attribution License 2.0, which permits unrestricted use, distribution, and reproduction in any medium, provided the original work is properly cited. 


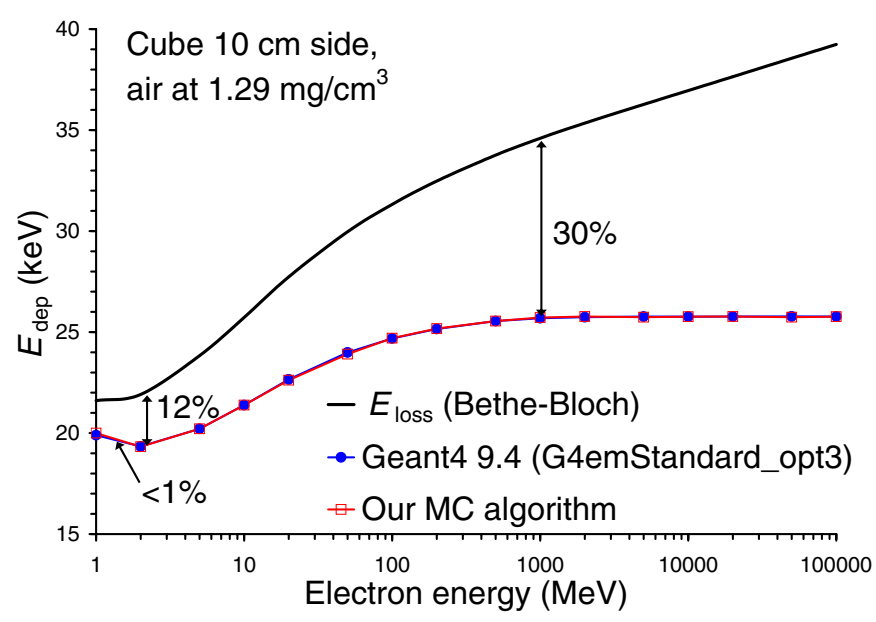

Figure 1. Comparison of results of electron energy deposition from our upgraded MC simulation and Geant 4 for a cube $10 \mathrm{~cm}$ side filled with air at $1.29 \mathrm{mg} / \mathrm{cm}^{3}$. An excellent agreement $(<1 \%)$ is observed in the wide energy range from $1 \mathrm{MeV}$ to $100 \mathrm{GeV}$. The energy loss given by the Bethe-Bloch formula is also shown to illustrate the contribution of escaping $\delta$ rays.

its impact on the determination of the absolute air-fluorescence yield in dedicated experiments. Results of energy deposition from an upgraded MC algorithm that we have developed are compared with those obtained using Geant4. Finally, corrections previously proposed in [4-6] to some fluorescence-yield measurements have been updated according to these calculations, and a reliable world average of the absolute air-fluorescence yield is presented.

\section{CALCULATION OF ENERGY DEPOSITION}

Collision stopping power is accurately described by the Bethe-Bloch theory, reference data being tabulated in [7] for a number of materials including air. However, for a correct determination of the energy deposited in a finite air volume, the fraction of energy transferred to secondary radiation escaping this volume has to be evaluated. In particular, high-energy secondary electrons ( $\delta$ rays) have large ranges. For instance, $100 \mathrm{keV}$ electrons can travel $14 \mathrm{~cm}$ in air at atmospheric pressure before being stopped. Although $\delta$ rays are barely produced, they carry a significant fraction of the energy lost by the primary particle. This effect causes the energy deposition to be lower than the total energy loss by $\sim 8 \%$ for incident electrons of $1 \mathrm{MeV}$ and by more than $30 \%$ for $\mathrm{GeV}$ electrons at usual experimental conditions (i.e., atmospheric pressure and a cm-sized collision chamber). This is illustrated in figure 1, where MC calculations of the energy deposition $E_{\text {dep }}$ in a $10 \mathrm{~cm}$ cube as a function of electron energy are plotted together with the Bethe-Bloch energy loss.

In addition, other features related to the propagation of electrons through the experimental collision chamber have minor effects on the actual energy deposition. Firstly, stopping power varies along the trajectories of electrons as they lose energy gradually. In the second place, scattering due to both elastic and inelastic collisions affects the average path length of electrons inside the considered volume, contributing to either a rise or a reduction of the energy deposition depending on geometry.

The energy deposition in the collision chamber of fluorescence-yield experiments is usually determined by available MC codes like Geant4 [8] and EGS4 [9]. We have developed an alternative MC algorithm $[1,10]$ using our own compilation of cross sections and other molecular data. Unlike standard MC codes, which normally employ the multiple scattering approximation, our program simulates all the individual interactions of electrons. Comparison of these independent simulations is thus a valuable test 

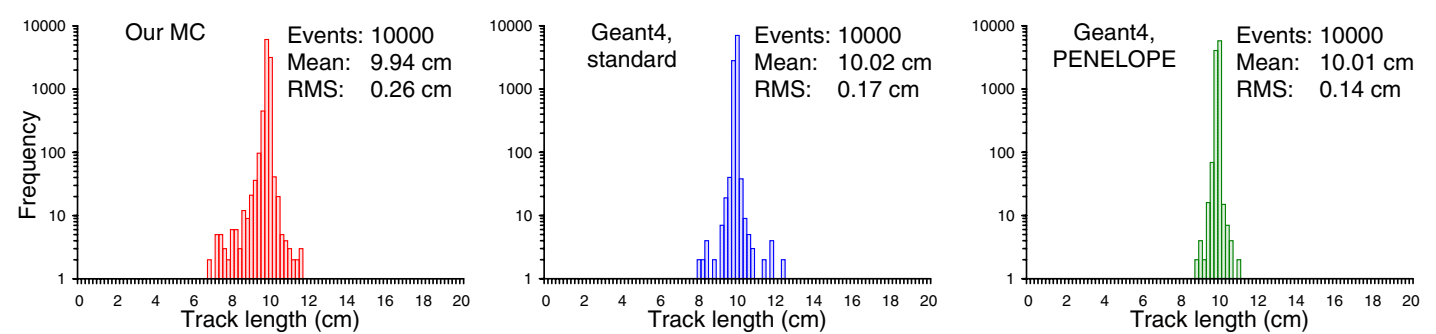

Figure 2. Track lengths of $1 \mathrm{MeV}$ electrons going through a $10 \mathrm{~cm}$ sphere (air at atmospheric pressure). From left to right, histograms are obtained using our MC algorithm, Geant 4 with the standard physics list and Geant 4 with the low-energy PENELOPE extension.

for the correctness and consistency of results on energy deposition, allowing estimation of uncertainties. This is mandatory to achieve high precision level $(\lesssim 5 \%)$ in the absolute fluorescence yield.

\section{COMPARISON OF MONTE CARLO SIMULATIONS}

A series of comparisons between our MC algorithm and Geant4 has been reported in previous works [4-6], showing general agreement. However, non-negligible discrepancies were found and they have been being investigated. As a result of these studies, several improvements have been made in our MC algorithm:

1. Corrections for the density effect in molecular cross sections, only noticeable at very high energy, have been reviewed and now are applied to the cross section for K-shell ionization as well (see [10] for details).

2. The energy distribution of secondary electrons has been modified to better reproduce the end tail of $\delta$ rays given by Möller scattering.

3. Angular-differential cross sections (both elastic and inelastic) have been corrected for some relativistic effects.

Updated results from our MC algorithm are presented here and compared with results from Geant4. In Figure 1, a comparison for the average energy deposition per electron in a cube of $10 \mathrm{~cm}$ side filled with air at $1.29 \mathrm{~g} / \mathrm{cm}^{3}(1013 \mathrm{hPa}$ and $273 \mathrm{~K})$ is shown. The geometry was defined such that electrons crossed the cube along an axis going through the center of opposite faces. For this comparison, the standard physics list G4emStandard_opt3 of Geant4 version 9.4, including multiple scattering, ionization, bremsstrahlung and emission of both $\mathrm{X}$ rays and Auger electrons, was used. An excellent agreement $(<1 \%)$ holds between both simulations in the wide energy range from $1 \mathrm{MeV}$ to $100 \mathrm{GeV}$. Different geometries and other physics lists of Geant4 have been tested, always showing excellent agreement.

In [6], we reported some minor disagreement ( $\sim 2 \%)$ between our MC algorithm and Geant4. They are mainly attributed to the inaccuracies in the energy distribution of secondaries in earlier versions of our code (improvement 2). The corrections in the angular-differential cross sections (improvement 3) turned out to be appreciable only at low energy $(\lesssim 1 \mathrm{MeV})$, since high-energy electrons follow almost rectilinear trajectories at those conditions. The latter corrections also contribute to a better agreement between both simulations.

On the other hand, we still find some differences between our MC algorithm and Geant4 in the simulated trajectories of electrons, which may be relevant at $\mathrm{keV}$ energies or in experiments with strong dependencies on geometrical factors. In Figure 2, histograms of track lengths of $1 \mathrm{MeV}$ electrons crossing a $10 \mathrm{~cm}$ diameter sphere filled with air at atmospheric pressure are shown for data from our upgraded MC algorithm and from Geant4 with the standard physics list or the low-energy PENELOPE 


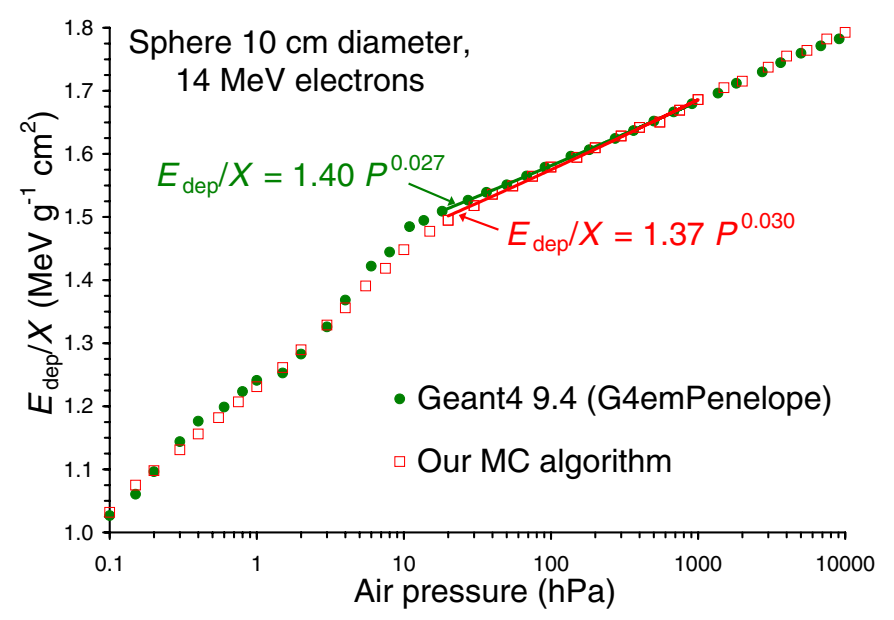

Figure 3. Comparison between our MC algorithm and Geant 4 for the pressure dependence of the energy deposition per unit mass thickness of air for $14 \mathrm{MeV}$ electrons crossing a $10 \mathrm{~cm}$ diameter sphere. Both simulations predict a power law with a marked change of slope at around $10 \mathrm{hPa}$. Best fits of data in the $15-1000 \mathrm{hPa}$ range are also shown.

extension (G4emPenelope library). Notice that data from our MC algorithm are more spread and its mean value is slightly lower than the predictions from both Geant4 simulations. These discrepancies could be due to the approximation of multiple scattering employed by Geant 4 and are under study.

Comparisons between our MC algorithm and Geant4 for the pressure dependence of the energy deposition have also been carried out. For this purpose, the energy deposited per unit mass thickness $\left(\mathrm{MeV} \mathrm{g}^{-1} \mathrm{~cm}^{2}\right)$ is calculated as the ratio of the average energy deposition and the average mass thickness $X$ traveled by incident electrons. Note that the trivial linear growth of the total energy loss with mass thickness vanishes in this ratio and it solely remains the pressure dependence due to the escaping energy carried by secondary radiation. This parameter is also insensitive to possible slight differences between the simulated track lengths of incident electrons. In Figure 3, results of the $E_{\text {dep }} / X$ ratio for $14 \mathrm{MeV}$ electrons crossing a $10 \mathrm{~cm}$ sphere are shown for the $0.1-10000 \mathrm{hPa}$ air pressure range $(293 \mathrm{~K})$. Very good agreement between both simulations is observed again, obtaining an approximated power law with a marked change of slope at around $10 \mathrm{hPa}$, which is associated to the average range of $410 \mathrm{eV}$ photons emitted by nitrogen after K-shell ionization (see [10] for a thorough interpretation of simulation results). In this comparison, the low-energy PENELOPE extension of Geant 4 has been used instead, as it allows to reduce the cut in energy for production of secondary radiation down to a value as low as $10 \mathrm{eV}$ (the lower the pressure, the lower the energy of secondary particles that can escape the sphere). The standard implementation of Geant4 gives very similar results at pressures above $15 \mathrm{hPa}$. Best fits of data in the $15-1000 \mathrm{hPa}$ interval (typical relevant pressures for cosmic-ray detection) are also shown in the figure.

It is worth mentioning that our Geant4 simulations are fully consistent with those performed by the MACFLY [12] and AIRFLY [11] experiments, which used this MC code to evaluate the energy deposition in their collision chambers. Some differences with respect to Geant 4 calculations made by the AirLight experiment [14] are found, nevertheless the authors of this experiment reported very recently [15] a bug in their simulation that likely justifies these discrepancies. Dedicated comparisons with other MC codes have not been performed yet, however tests have also been made $[4,5]$ using EGS4 data given by the FLASH experiment for $28.5 \mathrm{GeV}$ electrons [13]. These calculations deviate more significantly from ours (up to $8 \%$, depending on pressure), although the discrepancies could fairly be explained by a possible inaccurate treatment in [13] of the density effect, which is very significant at such an energy. Geometrical factors related to the spatial distribution of energy deposition in this 
UHECR 2012
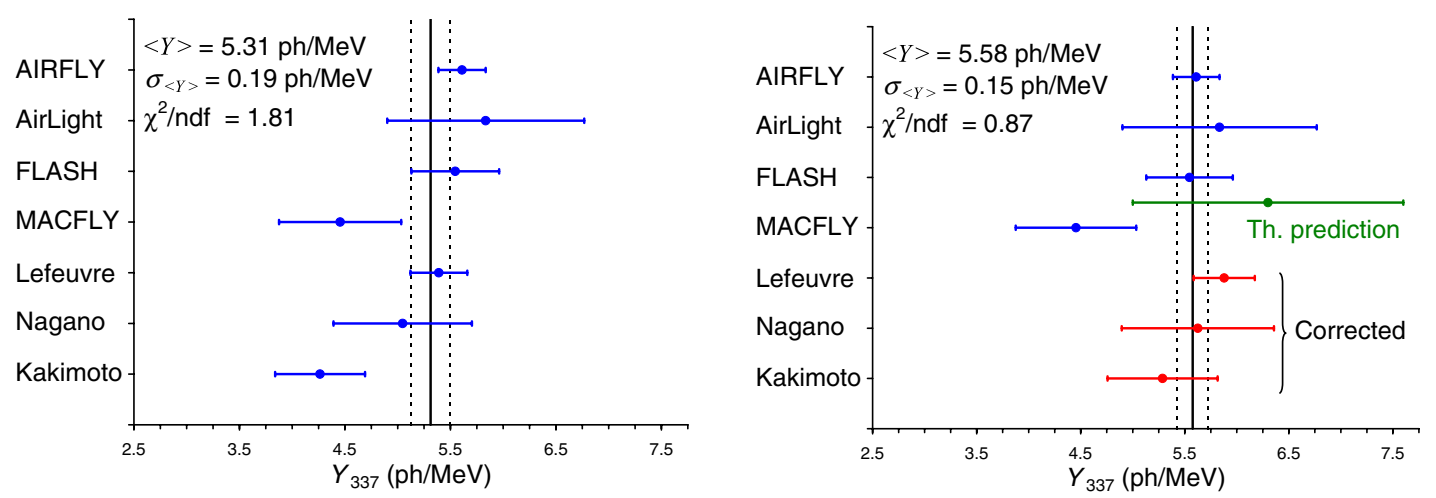

Figure 4. Effect of our corrections on the available fluorescence-yield measurements normalized to common conditions (1013 hPa, $293 \mathrm{~K}$ and $337 \mathrm{~nm}$ band). Original results are on the left and corrected ones (when applicable) are on the right, showing better compatibility. Vertical lines represent the position of the sample weighted average $\langle Y\rangle$ and its standard error $\sigma_{\langle Y\rangle}$. The theoretical fluorescence-yield value predicted by our MC simulation is also shown for comparison.

experiment have also been investigated, finding some discrepancies between the simulations that are still unclear.

Taking into consideration that our MC algorithm and Geant4 use different approaches for particle transport and are based on nearly independent molecular data, this consistency of results suggests that a high-precision level in the energy deposition is reached. On the other hand, this precision is limited by the accuracy in reference data of the Bethe-Bloch stopping power, which is estimated to be about $1 \%$ in the energy range of interest [7]. In view of that, we estimate a conservative total uncertainty of $2 \%$ in the above calculations of energy deposition.

\section{IMPACT ON THE FLUORESCENCE YIELD}

Several absolute measurements of the air-fluorescence yield are available in the literature. In recent experiments $[12-14,16]$, energy deposition was carefully evaluated by means of detailed Geant4 or EGS4 simulations. However, the authors of other well-known experiments [17-19] determined it from the Bethe-Bloch formula ignoring the effect of the escaping secondary radiation, which can cause large systematic errors, as discussed above.

In previous works [4-6], we proposed corrections to the energy deposition calculated in some of these experiments and the fluorescence-yield values were re-normalized correspondingly (the experimental fluorescence intensities were unchanged). As a consequence of these corrections, a very consistent sample of fluorescence-yield values was obtained, allowing us to calculate a reliable world average of this important parameter. The above-mentioned upgrades in our MC algorithm slightly affect this average. Next, updated results from this analysis are presented.

Figure 4 shows a comparison of the available experimental data of the absolute fluorescence yield normalized to common conditions, i.e., $337 \mathrm{~nm}$ band, dry air at $1013 \mathrm{hPa}$ and $293 \mathrm{~K}^{1}{ }^{1}$ Original fluorescence yields (after being normalized to these reference conditions) are plotted on the left. In the right plot, results from experiments that did not evaluate the energy deposition by simulation are corrected according to our calculations. Experimental uncertainties are not modified in any case,

\footnotetext{
${ }^{1}$ Quenching data as well as intensities of the remaining bands relative to the $337 \mathrm{~nm}$ band necessary for this normalization have been taken from [11].
} 


\section{EPJ Web of Conferences}

and it has been assumed that different measurements of a same experiment (only mean values are shown in 4) are fully correlated but no correlation exists between experiments. The sample includes the very precise measurement recently reported by the AIRFLY Collaboration [16], that use Geant 4 to evaluate the energy deposition assuming an error contribution of $2 \%$ to the total uncertainty of $4 \%$.

Our corrections increase the fluorescence yields of [17-19] by $9-24 \%$, leading to an improved compatibility of results. Notice that the $\chi^{2}$ statistic normalized by the number of degrees of freedom is lowered from 1.81 to 0.87 after our corrections. The sample average $\langle Y\rangle=5.58 \mathrm{ph} / \mathrm{MeV}$ is calculated using as weights the reciprocals of the squared experimental uncertainties. Somewhat different averages ranging from $5.46 \mathrm{ph} / \mathrm{MeV}$ to $5.68 \mathrm{ph} / \mathrm{MeV}$ are derived from plausible variations on the statistical analysis (e.g., treatment of outliers, weights and correlations) and on our corrections (e.g., geometrical implementation details in our simulations). The standard error of the weighted mean $\sigma_{\langle Y\rangle}=0.15 \mathrm{ph} / \mathrm{MeV}(2.6 \%)$ is determined from the quoted uncertainties following the usual procedure. However, this low uncertainty seems to us to be unrealistic, because some experiments did not include any error contribution from the evaluation of the energy deposition, which we estimate to be $2 \%$ at least for Geant 4 and our MC algorithm. In addition, there are still some discrepancies between simulations to be elucidated, as pointed out above. Taking into account all these considerations, we propose an average fluorescence-yield value of $5.58 \mathrm{ph} / \mathrm{MeV}$ and a conservative uncertainty estimate of $4 \%$, i.e., as low as the smallest uncertainty of the sample corresponding to the AIRFLY measurement. Indeed, it can be understood from the present analysis that this average and the AIRFLY measurement $(5.61 \mathrm{ph} / \mathrm{MeV})$ are complementary results showing high reliability.

Our MC algorithm is able to simulate both the energy deposition and the fluorescence emission $[1,10]$, which in combination with experimental quenching data from [11] also allows us to give a prediction of the absolute fluorescence yield of $6.3 \pm 1.3 \mathrm{ph} / \mathrm{MeV}$, in agreement with experimental data (Fig. 4, right plot). Although we estimate a large uncertainty in this value due to the uncertainties in the many molecular parameters involved in the calculation, the agreement with the experimental results provides a valuable theoretical support to these measurements.

\section{CONCLUSIONS}

We show that effects of secondary radiation escaping the collision chamber are very relevant for a correct determination of the energy deposition in experiments dedicated to measure the air-fluorescence yield. Assuming the Bethe-Bloch stopping power instead of computing the energy deposition by simulation may lead to large systematic errors in the fluorescence yield.

New results of energy deposition from an upgraded MC algorithm that we have developed are presented here, showing an excellent agreement with Geant 4 for electrons with energies in the wide $1 \mathrm{MeV}-100 \mathrm{GeV}$ range. From the present comparison, we conclude that these calculations have a precision level of $2 \%$. On the other hand, small differences in the simulated electron trajectories are found, with possible implications at lower energy or in experiments with strong dependencies on geometrical factors.

Our updated calculations of energy deposition have been used to correct experimental fluorescenceyield values reported by experiments that did not evaluate the energy deposition by simulation. These corrections improve significantly the compatibility of the available measurements of the absolute air-fluorescence yield. As a result of this analysis, we present a reliable average of $5.58 \mathrm{ph} / \mathrm{MeV}$ with an estimated uncertainty of $4 \%$. In addition, experimental data are shown to be consistent with a theoretical prediction of the absolute fluorescence yield that we obtained by means of our MC simulation.

This work has been supported by the Spanish Ministerio de Ciencia e Innovación (FPA2009-07772 and CONSOLIDER CPAN CSD2007-42) and "Comunidad de Madrid" (Ref.: 910600). 


\section{UHECR 2012}

\section{References}

[1] F. Arqueros et al., New J. Phys. 11 (2009) 065011

[2] J.W. Belz et al. [FLASH Collaboration], Astropart. Phys. 25 (2006) 57

[3] M. Ave et al. [AIRFLY Collaboration] Nucl. Instr. and Meth. A 597 (2008) 46

[4] J. Rosado, F. Blanco and F. Arqueros, Astropart. Phys. 34 (2010) 164

[5] J. Rosado, F. Blanco and F. Arqueros, AIP Conf. Proc. 1367 (2011) 34

[6] J. Rosado et al., 32nd International Cosmic Ray Conference (Beijing, 2011)

[7] A. Allisy et al., Stopping Powers for Electrons and Positrons (ICRU Report No. 37, 1984)

[8] S. Agostinelli et al., Nucl. Instrum. Meth. A 506 (2003) 250

[9] W.R. Nelson, H Hiragrama and D.W.O. Rogers, The EGS4 Code System (Stanford Linear Accelerator Center, SLAC-265, 1985)

[10] J. Rosado, PhD Thesis (2011)

[11] M. Ave et al. [AIRFLY Collaboration], Astropart. Phys. 28 (2007) 41

[12] P. Colin et al. [MACFLY Collaboration], Astropart. Phys. 27 (2007) 317

[13] R. Abbasi et al. [FLASH Collaboration], Astropart. Phys. 29 (2008) 77

[14] T. Waldenmaier et al., Astropart. Phys. 29 (2008) 205

[15] T. Waldenmaier et al., 8th Air-Fluorescence Workshop (Karlsruhe, 2011)

[16] M. Bohacova et al. [AIRFLY Collaboration], 8th Air-Fluorescence Workshop (Karlsruhe, 2011)

[17] F. Kakimoto et al., Nucl. Instrum. Meth. A 372 (1996) 205

[18] M. Nagano et al., Astropart. Phys. 22 (2004) 235

[19] G. Lefeuvre et al., Nucl. Instrum. Meth. A 578 (2007) 78205 